\title{
A Novel Mutation in the Arylsulfatase A Gene Associated with Adult-Onset Metachromatic Leukodystrophy without Clinical Evidence of Neuropathy
}

\author{
Chieko Suzuki $^{a}$ Mitsunori Watanabe ${ }^{a}$ Masahiko Tomiyama ${ }^{a}$ Kazuhiro Sugimoto ${ }^{b}$ \\ Eiji Nanbac Mandy Jackson ${ }^{d}$ Tamaki Kimura $^{a}$ Yusuke Seino ${ }^{a}$ Yasuhito Wakasaya ${ }^{a}$ \\ Takeshi Kawarabayashi $^{\mathrm{a}}$ Yasuo Miki ${ }^{\mathrm{a}}$ Yukiko Yamamoto-Watanabe ${ }^{\mathrm{a}}$ Mikio Shoji $^{\mathrm{a}}$ \\ a Department of Neurology, Institute of Brain Science, ${ }^{b}$ Department of Laboratory Medicine, \\ Hirosaki University Graduate School of Medicine, Hirosaki, and ${ }^{\mathrm{C} C e n t e r ~ f o r ~ B i o s c i e n c e ~ a n d ~ T e c h n o l o g y, ~}$ \\ Tottori University, Yonago, Japan; ${ }^{\mathrm{d}}$ Centre for Neuroscience, University of Edinburgh, Edinburgh, UK
}

Dear Sir,

Metachromatic leukodystrophy (MLD, MIM 250100) is an autosomal recessive hereditary disease characterized by neurological symptoms such as cognitive and behavioral abnormalities, ataxia and seizures [1]. MLD results from deficiency of arylsulfatase A (ASA), resulting in sulfatide accumulation in the central and peripheral nervous system [1]. A number of mutations in the ASA gene associated with decreased activity of ASA have been reported in patients with MLD [1]. Here we report a male patient with adult-onset MLD who is compound heterozygous for a novel nonsense mutation (C38X) $(\mathrm{TGC} \rightarrow \mathrm{TGA})$ and a known missense mutation (T409I) (ACT $\rightarrow$ ATT) $[2,3]$. Of note, this patient showed severely decreased nerve conduction velocities without obvious external clinical signs of neuropathy, normally a characteristic feature of MLD. The absence of clear external signs contributed to initial misdiagnosis.

The 33-year-old male developed irritability and disorientation and visited a hospital department of psychiatry. There he was diagnosed with schizophrenia and, although he continued to take medications appropriate to the diagnosis, his symptoms progressed. At age 34 his behavioral abnormalities worsened, and he was referred to our department. He was alert, but cognitively impaired, and his verbal, performance and full-scale scores on the Wechsler Adult Intelligence Scale-Revised were 57, $<45$ and 47 , respectively. His parents were not consanguineous and there is no previous history of similar neurological diseases in the family. Examination of the optic fundi showed no abnormal findings. He had no numbness, paresthesia, muscle weakness, muscle atrophy, abnormal involuntary movements or ataxia. Superficial sensation was normal, but vibratory sense was very slightly impaired in the lower limbs. The deep tendon reflexes were normal in the upper limbs. The patellar tendon reflexes were bilaterally increased, and the bilateral Achilles tendon reflexes were mildly diminished. The plantar responses were bilaterally extensor, but no spasticity was observed. Brain MRI revealed severe symmetrical white matter hyperintensities in the periventricular area on T2-weighted images and cerebral atrophy. Results from nerve conduction studies showed severely decreased motor and sensory nerve velocities (table 1). Semithin sections of sural nerve biopsy demonstrated mild loss of myelinated fibers and metachromatic deposits in the cytoplasm of Schwann cells and macrophages. On ultrastructural study, lamellar structures were present in the cytoplasm of Schwann cells. The ASA activity was $15.0 \mathrm{nmol} / \mathrm{mg}$ protein/h (normal range is $109-217 \mathrm{nmol} / \mathrm{mg}$ protein/h) in leukocytes. Blood samples were obtained from the patient with informed consent and genomic DNA was extracted by a standard method. All 8 exons of the ASA gene were amplified by polymerase chain reaction as described previously and sequenced [1]. The patient was found to be compound heterozygous for a novel nonsense mutation (C38X) $(\mathrm{TGC} \rightarrow \mathrm{TGA})$ and a known missense mutation (T409I) (ACT $\rightarrow$ ATT) resulting in a diagnosis of adult-onset MLD [2, 4].

Based on the age at onset, severity and progression of the disease, MLD is classified into 4 subtypes: late infantile, early juvenile, late juvenile and adult forms. Adultonset MLD is rare, and often presents with personality changes or cognitive deficits.

\section{KARGER}

Fax +41613061234 E-Mail karger@karger.ch www.karger.com
Dr. Mitsunori Watanabe

Department of Neurology, Institute of Brain Science

Hirosaki University Graduate School of Medicine

5 Zaifu-cho, Hirosaki 036-8562 (Japan)

Tel. +81 17239 5142, Fax +81 17239 5143, E-Mail miwata36@cc.hirosaki-u.ac.jp 
Therefore adult-onset MLD is sometimes misdiagnosed as schizophrenia, as was the case with this patient. It has been suggested that there is an association between activity of ASA and clinical subtype, with patients with a lower activity of ASA tending to have a younger age of onset. However, the activity of leukocyte ASA does not necessarily correlate with the clinical course of an individual [5].

The genotype-phenotype correlation is based on 2 functionally different types of mutations: mutations encoding inactive ASA (0 alleles) and mutations encoding ASA with residual enzymatic activity ( $R$ alleles) [6]. While genotypes consisting of two 0 alleles are generally associated with the severe late infantile type of MLD, combinations of $0 / R$ or $R / R$ genotypes tend to lead to juvenile or adult forms. In this patient, the activity of ASA was reduced to approximately $10 \%$ of the normal range, indicating that the truncated ASA protein of 37 amino acids occurring due to the novel mutation, C38X (TGC $\rightarrow$ TGA) has no activity of ASA (0 allele), since the T409I mutation (ACT $\rightarrow$ ATT) is known to be an $\mathrm{R}$ allele [2]. Therefore, in this patient a $0 / R$ combination of alleles results in a loss of approximately $90 \%$ of ASA protein activity and adult-onset MLD. Although severely decreased nerve conduction velocity was observed in this patient, there was no clinical evidence of peripheral neu-

Table 1. Results of nerve conduction studies

\begin{tabular}{lllll}
\hline Motor & $\begin{array}{l}\text { Amplitude } \\
\text { (distal/proximal), } \mathrm{mV}\end{array}$ & $\begin{array}{l}\text { Distal } \\
\text { latency, ms }\end{array}$ & $\begin{array}{l}\mathrm{MCV} \\
\mathrm{m} / \mathrm{s}\end{array}$ & $\begin{array}{l}\text { F latency } \\
\mathrm{ms}\end{array}$ \\
\hline Median & $10 / 10$ & 5.7 & 22 & 49 \\
Ulnar & $17 / 13$ & 5.9 & 22 & 50 \\
Tibial & $10 / 3$ & 6.8 & 20 & 88 \\
\hline Sensory & Amplitude & SCV & \\
& (wrist/elbow), $\mu \mathrm{V}$ & (wrist/elbow), $\mathrm{m} / \mathrm{s}$ & \\
\hline Median & $3 / 3$ & $24 / 27$ & & \\
Ulnar & $4 / 2$ & $20 / 24$ & \\
Sural & not evoked & not evoked & \\
& & &
\end{tabular}

$\mathrm{MCV}=$ Motor nerve conduction velocity; SCV = sensory nerve conduction velocity.

ropathy, despite peripheral neuropathy, in addition to leukodystrophy, being a characteristic feature of MLD. It may be that the absence of clinically evident peripheral neuropathy was due to the neurological examination being carried out shortly after the onset of the disease. Nevertheless this case highlights that MLD must still be strongly considered as a diagnosis in patients presenting with personality or cognitive alterations, but lacking clinical symptoms of neuropathy.

\section{Acknowledgements}

The authors thank Drs. Masayuki Baba, Akira Arai and Yoshio Ikeda for their kind help. This study was supported by Grants-in-Aid for the Primary Amyloidosis Research Committee (M. Yamada), from the Ministry of Health, Labor and Welfare of Japan, by the grant provided by Sapporo Biocluster 'Bio-S', the Knowledge Cluster Initiative of the MEXT and by Grants-in-Aid for Scientific Research (B) (19390233).

\section{References}

1 Scriver CR, Beaudet AL, Sly WS, Val D (eds): The Metabolic and Molecular Bases of Inherited Disease, ed 8. New York, McGrawHill, 2001.

$\checkmark 2$ Hasegawa Y, Kawame H, Ida H, Ohashi T, Eto Y: Single exon mutation in arylsulfatase A gene has two effects: loss of enzyme activity and aberrant splicing. Hum Genet 1994; 93:415-420.
-3 Fukutani Y, Noriki Y, Sasaki K, Isaki K, Kuriyama M, Kurosawa K, Ida H: Adult-type metachromatic leukodystrophy with a compound heterozygote mutation showing character change and dementia. Psychiatry Clin Neurosci 1999;53:425-428.

4 Kurosawa K, Ida H, Eto Y: Prevalence of arylsulphatase A mutations in 11 Japanese patients with metachromatic leukodystrophy: identification of two novel mutations. J Inherit Metab Dis 1998;21:781-782.

5 Kreysing J, Bohne W, Bosenberg C, Marchesini S, Turpin JC, Baumann N, von Figura K, Gieselmann V: High residual arylsulfatase A (ARSA) activity in a patient with late-infantile metachromatic leukodystrophy. Am J Hum Genet 1993;53:339-346.
6 Rauschka H, Colsch B, Baumann N, Wevers R, Schmidbauer M, Krammer M, Turpin JC, Lefevre M, Olivier C, Tardieu S, Krivit W, Moser H, Moser A, Gieselmann V, Zalc B, Cox T, Reuner U, Tylki-Szymanska A, AboulEnein F, LeGuern E, Bernheimer H, Berger J: Late-onset metachromatic leukodystrophy: genotype strongly influences phenotype. Neurology 2006;67:859-863. 